\title{
Intrapartum Group B Streptococcus Antibiotic Prophylaxis in Penicillin Allergic Pregnant Women
}

\author{
Nerlyne Desravines, $\mathrm{MD}^{1} \quad$ Kartik K. Venkatesh, MD/PhD ${ }^{1}$ Austin Hopkins, $\mathrm{MS}^{1} \quad$ Jamie Waldron, MD ${ }^{2}$ \\ Megan Grant, MS ${ }^{1}$ Colleen McGuire, MS ${ }^{1} \quad$ Kim A. Boggess, MD ${ }^{1}$ \\ ${ }^{1}$ Division of Maternal Fetal Medicine, Department of Obstetrics and \\ Gynecology, The University of North Carolina at Chapel Hill, Chapel \\ Hill, North Carolina \\ 2 Division of Rheumatology, Allergy and Immunology, Department of \\ Internal Medicine, The University of North Carolina at Chapel Hill, \\ Address for correspondence Nerlyne Desravines, MD, Division of \\ Maternal-Fetal Medicine, Department of Obstetrics and Gynecology, \\ The University of North Carolina at Chapel Hill, 3031 Old Clinic \\ Building CB \#7570, Chapel Hill, NC 27599 \\ (e-mail: nerlyne.desravines@med.unc.edu).
} Chapel Hill, North Carolina

Am J Perinatol Rep 2019;9:e238-e243.

\begin{abstract}
Keywords

- GBS prophylaxis

- penicillin allergy

- cephalosporin allergy

Objectives To estimate the prevalence of and identify modifiable risk factors for alternative antibiotics for group B Streptococcus (GBS) prophylaxis in penicillin-allergic women. Methods Retrospective cohort study of pregnant women within a health care network from January 1, 2014, to December 31, 2017. Included women were GBS colonized, delivered at $\geq 37$ weeks' gestation, and reported penicillin/cephalosporin allergy. The primary outcome was the use of alternate antibiotics GBS prophylaxis, defined per Centers for Disease Control and Prevention guidelines as antibiotics other than penicillin, ampicillin, or cefazolin.

Results We identified 190 GBS-colonized pregnant women self-reporting a penicillin/ cephalosporin allergy; 5\% reported anaphylaxis, 44\% high-risk symptoms (isolated hives, shortness of breath, swelling, or vomiting), and $51 \%$ low-risk symptoms (isolated rash, itching, or nausea). Two-thirds (63\%) had alternative antibiotic prophylaxis. In adjusted analyses, nonwhite race (adjusted odds ratio [aOR]: 2.42; 95\% confidence interval [Cl]: 1.19-4.94) and high-risk allergic reaction (aOR: 2.42; 95\% Cl: 1.30-4.49) were associated with higher odds of alternative antibiotics prophylaxis compared with low-risk allergic reaction. Low-risk allergic reaction group was less likely to receive alternative antibiotic prophylaxis (aOR: 0.36; $95 \mathrm{Cl} \%$ : 0.19-0.66).

Conclusion Alternative antibiotic use for GBS prophylaxis is frequent with penicillin/ cephalosporin allergies. Efforts to confirm allergy and perform penicillin hypersensitivity testing may increase compliance with guidelines for antibiotic administration.
\end{abstract}

Group B Streptococcus (GBS) is a bacterium that can colonize the genitourinary tract of pregnant women and is a leading cause of early-onset neonatal sepsis via vertical transmission. ${ }^{1}$ Nearly one in five U.S. pregnant women are GBS colonized and the risk of vertical transmission at delivery is as high as $50 \%{ }^{2}$ In 1996 , (1D) Kartik Venkatesh's ORCID is https://orcid.org/0000-0002-8043-
556X.

received

January 10, 2019

accepted after revision

April 19, 2019
DOI https://doi.org/

$10.1055 / \mathrm{s}-0039-1694031$. ISSN 2157-6998. the Centers for Disease Control and Prevention (CDC) recommended third trimester maternal GBS screening and intrapartum antibiotic treatment with penicillin to reduce risk of early-onset neonatal GBS sepsis. GBS is susceptible to $\beta$-lactam antibiotics, including ampicillin, penicillin, and cephalosporins. With a reported penicillin allergy, alternate antibiotics include cefazolin, erythromycin, or clindamycin. ${ }^{3}$ However, based on previous guidelines, the use of erythromycin and clindamycin

Copyright @ 2019 by Thieme Medical Publishers, Inc., 333 Seventh Avenue, New York, NY 10001, USA. Tel: +1(212) 584-4662.
License terms

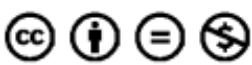


required previous GBS culture with antibiotic susceptibility testing due to variable coverage and resistance. ${ }^{3}$ Susceptibility testing is an antimicrobial resistance panel that analyses the sensitivity of antibiotics, including clindamycin, to GBS.

According to 2010 CDC guidelines, only intrapartum treatment with penicillin and cefazolin is considered optimal for GBS prophylaxis to reduce risk for early-onset GBS sepsis. ${ }^{3,4}$ Erythromycin has subsequently been removed as an alternate antibiotic option in the setting of an allergy due to increasing resistance. Multiple studies have shown poor adherence to these guidelines, particularly in utilizing cefazolin as an alternative treatment option in penicillin allergic pregnant women. ${ }^{5,6}$ These studies have been primarily at single-site academic institutions and only one study assessed optimal antibiotic prophylaxis using the updated 2010 CDC guidelines. Modifiable factors to improve adherence to the 2010 CDC guidelines have not yet been identified.

Approximately $10 \%$ of the general population report a penicillin allergy, with less than $1 \%$ reporting a severe reaction such as anaphylaxis. ${ }^{7}$ Penicillin allergy can be evaluated using skin testing and current penicillin tolerance was confirmed using an oral therapeutic dose of amoxicillin and 1 hour of observation. ${ }^{8,9}$ Penicillin allergy evaluations have been shown to be safe in pregnancy, with a very low risk of serious testingassociated reaction. ${ }^{8}$ Additionally, prior data also suggest that the majority of pregnant women colonized with GBS with a self-reported penicillin allergy are confirmed to be currently tolerant to penicillin. ${ }^{10}$

The primary objective of this study was the use of optimal antibiotics GBS prophylaxis, defined per CDC guidelines as antibiotics use of penicillin, ampicillin, or cefazolin. Additionally, we wanted to identify potentially modifiable risk factors for optimal antibiotic prophylaxis. We defined modifiable risk factors as clinical factors that may influence the choice of antibiotic administered, such as the type of provider, level of training, site of prenatal care, and primary language. Applying the 2010 CDC guidelines to measure prevalence across a health care system adds the current body of knowledge as it allows us to examine practice across multiple practice settings including private practice, midwifery, academic practice, and resident clinics.

\section{Methods}

We conducted a retrospective cohort study of pregnant women receiving antenatal care and infant delivery at the University of North Carolina health care system between January 1, 2014, and December 31, 2017. We queried the electronic medical record (EMR) to identify all deliveries complicated by maternal genitourinary GBS colonization, defined as: (1) a positive rectovaginal swab culture, (2) positive urine culture, and/or (3) a positive test result noted in the results console of the EMR. We reviewed the medical records of 1,292 GBS-colonized women and identified 270 (21\%) with a self-reported penicillin, ampicillin, or cephalosporin allergy. Of these 270 women, 190 were delivered at term ( $\geq 37$ weeks) in the absence of chorioamnionitis or other indication for antibiotic use in labor. The current analysis is limited to these 190 term deliveries (- Fig. 1). This study was approved by the Institutional Review Board at the University of North Carolina, Chapel Hill.

We abstracted maternal demographic, medical, and obstetric characteristics, and site of prenatal care (private practice, midwifery care, family medicine, academic attending clinic, and resident clinic). Modifiable risk factors in this context refer to clinical factors that may influence the choice of antibiotic such as the type of provider (midwife vs. medical doctor), level of training, site of prenatal care (academic tertiary care center or private community hospital), and primary language of the patient which serves as a proxy for a language barrier. The maternal characteristics that were utilized for comparison between the women who received optimal and alternative antibiotic therapies are listed in - Table 1.

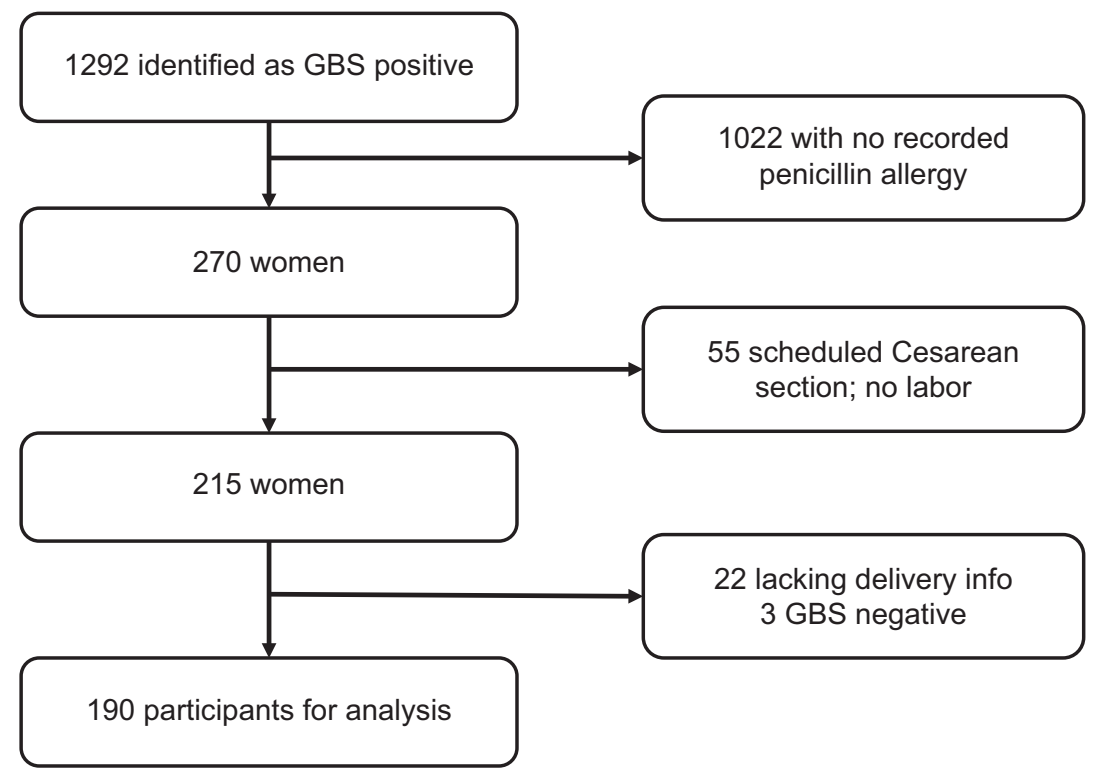

Fig. 1 Schema for subject inclusion and exclusion for analysis. 
Table 1 Baseline patient characteristics of term GBS-colonized pregnant women with a self-reported penicillin allergy by intrapartum antibiotic treatment status $(n=190)$

\begin{tabular}{|c|c|c|c|}
\hline & $\begin{array}{l}\text { Whole cohort } \\
(n=190)\end{array}$ & $\begin{array}{l}\text { Alternative antibiotic } \\
\text { treatment }(n=119)\end{array}$ & $\begin{array}{l}\text { Optimal antibiotic } \\
\text { treatment }(n=71)\end{array}$ \\
\hline Age (y), median (range) & $31(15-44)$ & $31(15-41)$ & $32(18-44)$ \\
\hline Nulliparous & $85(45 \%)$ & $50(42 \%)$ & $35(49 \%)$ \\
\hline Race, Caucasian & $113(59 \%)$ & $70(59 \%)$ & $55(77 \%)$ \\
\hline BMI $\left(\mathrm{kg} / \mathrm{m}^{2}\right)$, median (range) & $29(19-55)$ & $29(19-55)$ & $29(20-54)$ \\
\hline Relationship status, married & $132(69 \%)$ & $76(64 \%)$ & $56(79 \%)$ \\
\hline Primary language, English & $183(96 \%)$ & $115(96 \%)$ & $68(96 \%)$ \\
\hline Insurance, private & $135(71 \%)$ & $83(70 \%)$ & $52(73 \%)$ \\
\hline \multicolumn{4}{|l|}{ Prenatal site } \\
\hline University-based attending clinic & $46(24 \%)$ & $25(21 \%)$ & $21(30 \%)$ \\
\hline University-based resident clinic & $20(11 \%)$ & $15(13 \%)$ & $5(7 \%)$ \\
\hline Community-based private practice & $119(63 \%)$ & $76(64 \%)$ & $43(60 \%)$ \\
\hline Community-based health center & $5(2 \%)$ & $3(2 \%)$ & $2(3 \%)$ \\
\hline \multicolumn{4}{|l|}{ Method of GBS status } \\
\hline Rectovaginal swab & $179(94 \%)$ & $113(95 \%)$ & $66(93 \%)$ \\
\hline Urine culture & $10(5 \%)$ & $5(4 \%)$ & $5(7 \%)$ \\
\hline Reported positive & $1(1 \%)$ & $1(1 \%)$ & - \\
\hline Susceptibilities obtained & $96(51 \%)$ & $70(59 \%)$ & $26(37 \%)$ \\
\hline \multicolumn{4}{|l|}{ Allergy $^{\mathrm{a}}$} \\
\hline Penicillin & $171(90 \%)$ & $112(94 \%)$ & $59(83 \%)$ \\
\hline Cephalosporin & $25(13 \%)$ & $15(13 \%)$ & $10(14 \%)$ \\
\hline \multicolumn{4}{|l|}{ Allergic reaction } \\
\hline Anaphylaxis & $10(5 \%)$ & $8(7 \%)$ & $2(3 \%)$ \\
\hline High risk for anaphylaxis & $84(44 \%)$ & $62(52 \%)$ & $22(31 \%)$ \\
\hline Low risk for anaphylaxis & $96(51 \%)$ & $49(41 \%)$ & 47 (66\%) \\
\hline \multicolumn{4}{|l|}{ Admission diagnosis } \\
\hline Spontaneous labor & $100(53 \%)$ & $64(54 \%)$ & $36(51 \%)$ \\
\hline Induction of Labor & $90(47 \%)$ & $55(46 \%)$ & $35(49 \%)$ \\
\hline \multicolumn{4}{|l|}{ Cervical dilation on admission } \\
\hline $0-6 \mathrm{~cm}$ & $161(85 \%)$ & $96(81 \%)$ & $63(89 \%)$ \\
\hline$>6 \mathrm{~cm}$ & $29(15 \%)$ & $23(19 \%)$ & $8(11 \%)$ \\
\hline Rupture on admission & $48(25 \%)$ & $32(27 \%)$ & $16(23 \%)$ \\
\hline \multicolumn{4}{|l|}{ Mode of delivery } \\
\hline Vaginal delivery & $158(83 \%)$ & $95(80 \%)$ & $63(89 \%)$ \\
\hline Cesarean delivery & $32(17 \%)$ & $24(20 \%)$ & $8(11 \%)$ \\
\hline \multicolumn{4}{|l|}{ Antibiotic used } \\
\hline Penicillin/ampicillin & $25(13 \%)$ & - & $25(35 \%)$ \\
\hline Cefazolin & $46(24 \%)$ & - & $46(65 \%)$ \\
\hline Clindamycin & $68(36 \%)$ & $68(57 \%)$ & - \\
\hline Vancomycin & $38(20 \%)$ & $38(32 \%)$ & - \\
\hline None & $11(6 \%)$ & $11(9 \%)$ & - \\
\hline Alternative antibiotics & $2(1 \%)$ & $2(2 \%)$ & - \\
\hline
\end{tabular}

Abbreviations: BMI, body mass index; GBS, group B Streptococcus.

${ }^{a}$ Not mutually exclusive. 
Table 2 Schema for classification of allergy symptom

\begin{tabular}{|l|l|l|}
\hline Anaphylaxis & $\begin{array}{l}\text { Allergic reaction } \\
\text { stated in the } \\
\text { medical record } \\
\text { as anaphylaxis }\end{array}$ & \\
\hline High risk & $\begin{array}{l}\text { Isolated } \\
\text { symptoms } \\
\text { which may be } \\
\text { associated with } \\
\text { anaphylaxis }\end{array}$ & $\begin{array}{l}\text { Hives or urticaria } \\
\text { Wheezing } \\
\text { Swelling } \\
\text { Vomiting }\end{array}$ \\
\hline Low risk & $\begin{array}{l}\text { Isolated } \\
\text { symptoms } \\
\text { not classically } \\
\text { associated with } \\
\text { anaphylaxis }\end{array}$ & $\begin{array}{l}\text { Nausea (without vomiting) } \\
\text { Itching (without } \\
\text { associated rash) } \\
\text { Rash (nonspecified } \\
\text { hives/urticarial) } \\
\text { Not otherwise specified } \\
\text { Unknown reactions }\end{array}$ \\
\hline
\end{tabular}

We categorized participants' reported drug allergy reactions into one of three groups: a severe reaction, high-risk allergic reaction, and low-risk allergic reaction. A severe reaction is defined as a history of anaphylaxis. A high risk for allergic reaction signifies an isolated symptom that may be associated with anaphylaxis however does not meet criteria for anaphylaxis. ${ }^{11,12}$ These symptoms included urticarial or hives, wheezing, swelling, and vomiting. We defined a reported reaction as a low-risk allergic reaction if the symptom was nonspecific or not suggestive of anaphylaxis. This includes nonspecific rash, itching in the absence of urticarial or rash, or nausea without vomiting. An allergy listed in the health record without an accompanying allergic reaction or reported unknown reaction was classified as a low-risk reaction for analytical purposes. This schema is outlined in - Table 2.

The primary outcome was the administration of alternate antibiotic therapy for GBS prophylaxis, defined per 2010 CDC guidelines as the use of intrapartum antibiotics other than penicillin, ampicillin, or cefazolin. ${ }^{3}$ We also determined the prevalence of testing for antibiotic susceptibility to GBS in our cohort, also a CDC recommendation.

We compared maternal characteristics between women with optimal and alternative antibiotic therapies for GBS prophylaxis. In univariate analyses, Student's $t$-test and chisquare test were used for continuous and categorical variables, respectively. We then used logistic regression to identify predictors of alternative antibiotic therapy for GBS prophylaxis. Based on the univariate analysis $(p<0.10)$, the multivariable analysis adjusted for maternal race and prior susceptibility testing during pregnancy. All analyses were conducted using STATA MP 15.1 (College Station, TX).

\section{Results}

- Table 1 shows maternal demographic and medical characteristics of the 190 term pregnant women who were GBS colonized anytime during pregnancy with a reported prior penicillin or cephalosporin allergy. The average age and body mass index was 30 years and $31 \mathrm{~kg} / \mathrm{m}^{2}$, respectively. The majority of women were Caucasian (65\%), privately insured (71\%), married (69\%), and English speaking (96\%). More than half $(52 \%)$ of the patients were delivered in a community hospital staffed by private attending physicians. The average gestational age at delivery was $38^{6 / 7}$ weeks. On admission, $52 \%$ were admitted with spontaneous labor, $72 \%$ intact, and $85 \%$ in latent labor. The average time of rupture to delivery was $\sim 7$ hours. Ten (5\%) reported a severe allergic reaction, 84 (43\%) reported a high-risk allergic reaction, and 96 (51\%) reported a low-risk allergic reaction. In patients reporting more than one symptom, the response was recorded for the higher of the two categories.

Seventy-one (37\%) of 190 were given optimal antibiotics, and 106 (56\%) were treated with alternative antibiotic therapy, most notably, clindamycin and vancomycin. There were 11 women (6\%) who did not receive any antibiotics and 2 (1\%) who received alternative antibiotics such as gentamicin and erythromycin. Only 97 (51\%) of 190 women had antibiotic susceptibility testing, and only 6 (60\%) of the 10 women with reported anaphylaxis underwent susceptibility testing.

In adjusted analyses, pregnant women of nonwhite race (adjusted odds ratio [aOR]: 2.42; 95\% confidence interval [CI]: 1.30-4.49) and with a high-risk allergic reaction (aOR: 2.49; 95\% CI: 1.33-4.65) were more likely to receive alternative antibiotic prophylaxis compared with those of white race or reported a low-risk allergic reaction or anaphylaxis (-Table 3). Women reporting a low-risk allergic reaction were less likely to receive alternative antibiotic prophylaxis (aOR: 0.39; 95\% CI: 0.20-0.77) than those with a high-risk allergic reaction or anaphylactic allergic reaction.

Table 3 Factors associated with alternate antibiotic prophylaxis for GBS among term pregnant women with a prior history of penicillin/cephalosporin allergy

\begin{tabular}{|c|c|c|}
\hline & $\begin{array}{l}\text { Unadjusted odds } \\
\text { ratio, } 95 \% \mathrm{Cl}\end{array}$ & $\begin{array}{l}\text { Adjusted odds }{ }^{a} \\
\text { ratio, } 95 \% \mathrm{Cl}\end{array}$ \\
\hline \multicolumn{3}{|l|}{ Race } \\
\hline White & Referent & Referent \\
\hline Nonwhite & $2.35(1.19-4.66)^{\mathrm{b}}$ & $2.42(1.19-4.94)^{b}$ \\
\hline \multicolumn{3}{|c|}{ Susceptibility testing } \\
\hline No & Referent & Referent \\
\hline Yes & $2.41(1.31-4.43)^{\mathrm{b}}$ & $2.31(1.23-4.37)^{\mathrm{b}}$ \\
\hline \multicolumn{3}{|l|}{ Anaphylaxis } \\
\hline No & Referent & Referent \\
\hline Yes & $2.46(0.51-12.05)$ & $1.55(0.28-8.33)$ \\
\hline \multicolumn{3}{|l|}{ High risk } \\
\hline No & Referent & Referent \\
\hline Yes & $2.42(1.30-4.49)^{\mathrm{b}}$ & $2.49(1.33-4.65)^{b}$ \\
\hline \multicolumn{3}{|l|}{ Low risk } \\
\hline No & Referent & Referent \\
\hline Yes & $0.35(0.19-0.65)^{\mathrm{b}}$ & $0.36(0.19-0.66)^{b}$ \\
\hline
\end{tabular}

Abbreviation: $\mathrm{Cl}$, confidence interval.

Note: Data presented as mean (standard deviation) or number (\%).

${ }^{a}$ Adjusted model controlled for race.

bStatistically significant finding with $p$-value $<0.05$. 


\section{Discussion}

Antibiotic treatment recommendations, including options for patients who report a penicillin or cephalosporin allergy, are published by the CDC and most recently updated in $2010 .^{3}$ Despite the guidelines set forth, in our cohort, pregnant women with a penicillin allergy were frequently receiving alternative GBS antibiotic prophylaxis. We found that two-thirds of GBScolonized term pregnant women with a reported penicillin/ cephalosporin allergy received alternative antibiotic GBS prophylaxis. Women with a low-risk allergic reaction were less likely to receive alternative antibiotics. We did not find an association between anaphylaxis and alternative antibiotic prophylaxis; however, this is likely due to the limited sample.

Though patients with a low-risk allergic reaction were less likely to receive alternative antibiotic prophylaxis, in our study group, more than half (53\%) of women with a low-risk allergic received alternative antibiotic treatment. The underlying reason for these associations remain unclear, but may include practice patterns of individualized practices and hospitals, availability of clinical pharmacist in community or academic centers, and the comfort and perception of the care provider. A survey of clinicians showed that only 68 to $80 \%$ of clinicians would prescribe a cephalosporin in a patient with previous maculopapular rash to penicillin. ${ }^{13}$ This may suggest provider discomfort with utilizing a cephalosporin with any previously reported history. Women of nonwhite race as well as those with a high-risk allergic reaction were more likely to have alternative antibiotic prophylaxis. The association between nonwhite rate and alternate antibiotic prophylaxis is of concern, and further efforts will be needed to ensure that minority women are receiving optimal prophylaxis, particularly because this is a population with overall higher rates of GBS colonization.

In this study, 10 women reported an anaphylactic reaction to penicillin or ampicillin, yet 2 patients received penicillin. The two women with an anaphylactic reaction to penicillin received penicillin for prophylaxis, which may have been used at the discretion of the provider after further investigation of the patient's prior allergic history, including the possibility that the patient may have completed allergy testing which was not captured in the EMR. Studies have shown that in the nonpregnant population, despite a $10 \%$ prevalence rate for penicillin allergies, only a small proportion has true penicillin allergy. ${ }^{7}$ Most participants in our study reported either low- or high-risk allergic reactions ( 60 and $43 \%$, respectively), but it was the high-risk group that was most likely to be treated with alternative antibiotics. In this group, elaboration of allergy history and further characterization of the associated reaction may have the most impact. Current studies suggest that penicillin allergy can wave over time, even in the setting of anaphylactic reactions. ${ }^{9}$

Clinically, the high-risk allergic reactions which signify symptoms that can be associated with anaphylaxis but does not define an anaphylactic reaction may be most difficult to assess for appropriate antibiotics coverage. Making a choice for antibiotics requires a more thorough evaluation of the allergy history including the exact reaction, time between exposure and reaction, subsequent antibiotic exposure, and length of time since last known reaction. It is these clarifications which can allow the clinician to determine if the patient's isolated symptoms suggest an immediate immunoglobulin E-mediated reaction that is consistent with anaphylaxis. ${ }^{11}$ In our electronic medical health record, these allergies are flagged as "high risk" in the patient chart, but in practice, patients often offer ambiguous nonspecific histories.

Antibiotic sensitivity testing was also evaluated during this study. Of the 190 women, only $62 \%$ had a sensitivity testing done. While clindamycin is not considered optimal antibiotic coverage in terms of the CDC 2010 guidelines, it does offer more tailored coverage when compared with vancomycin. With our limited sample size, correlations between increased sensitivity testing and subsequent clindamycin administration are hard to make; however, it remains evident that sensitivity-testing rates are low.

Strengths in this study include the broad range of obstetric providers which are encompassed within the single health care system. These include private attendings, academic attendings, midwives, residents, and nurse practitioners. The antenatal period may be the most appropriate time to further explore a drug allergy, and it is these providers that determine which patients require susceptibility testing. Our data show that obstetric providers need to improve guideline-based compliance for GBS prophylaxis and susceptibility testing. A limitation of this study was its retrospective nature. While delivery data and antibiotic use were recorded for each participant, any further elaboration of the patient's allergy, timing and setting of the patient's previous allergy, previous antibiotic exposure, and patient comfort with using optimal antibiotic prophylaxis could not be captured from chart review. Additionally, the provider's perception on the risk of harm could not be captured given this was a retrospective analysis based on chart abstraction. Also, limitation in this dataset is specific antibiotic reactions and history of prior skin testing.

In conclusion, the use of alternative antibiotics for GBS prophylaxis is frequent among term women with reported penicillin/cephalosporin allergy. Antibiotic susceptibility testing in the antepartum period was also found to be inadequate. Of note, obstetric providers do use penicillin and cephalosporins for GBS prophylaxis in women who report low-risk allergic reactions, mirroring $\mathrm{CDC}$ guidelines. Efforts to confirm the severity of prior antibiotic allergic reactions and perform penicillin sensitivity testing have the potential to increase guideline compliant antibiotic administration in this population.

\section{Conflict of Interest \\ None.}

\section{Acknowledgments}

Data collection utilized Research Electronic Data Capture (REDCap). The project described was supported by the National Center for Advancing Translational Sciences (NCATS), National Institutes of Health, through grant award number UL1TR001111. The content is solely the responsibility of the authors and does not necessarily represent the official views of the $\mathrm{NIH}$. 


\section{References}

1 Schuchat A. Epidemiology of group B streptococcal disease in the United States: shifting paradigms. Clin Microbiol Rev 1998;11 (03):497-513

2 Regan JA, Klebanoff MA, Nugent RP; Vaginal Infections and Prematurity Study Group. The epidemiology of group B streptococcal colonization in pregnancy. Obstet Gynecol 1991;77(04):604-610

3 Verani JR, McGee L, Schrag SJ; Division of Bacterial Diseases, National Center for Immunization and Respiratory Diseases, Centers for Disease Control and Prevention (CDC). Prevention of perinatal group B streptococcal disease-revised guidelines from CDC, 2010. MMWR Recomm Rep 2010;59(RR-10):1-36

4 Pearlman MD, Pierson CL, Faix RG. Frequent resistance of clinical group B streptococci isolates to clindamycin and erythromycin. Obstet Gynecol 1998;92(02):258-261

5 Paccione KA, Wiesenfeld HC. Guideline adherence for intrapartum group B streptococci prophylaxis in penicillin-allergic patients. Infect Dis Obstet Gynecol 2013;2013:917304

6 Briody VA, Albright CM, Has P, Hughes BL. Use of cefazolin for group $B$ streptococci prophylaxis in women reporting a penicillin allergy without anaphylaxis. Obstet Gynecol 2016;127(03):577-583
7 Albin S, Agarwal S. Prevalence and characteristics of reported penicillin allergy in an urban outpatient adult population. Allergy Asthma Proc 2014;35(06):489-494

8 Macy E. Penicillin skin testing in pregnant women with a history of penicillin allergy and group B Streptococcus colonization. Ann Allergy Asthma Immunol 2006;97(02):164-168

9 Bhattacharya S. The facts about penicillin allergy: a review. J Adv Pharm Technol Res 2010;1(01):11-17

10 Philipson EH, Lang DM, Gordon SJ, Burlingame JM, Emery SP, Arroliga ME. Management of group B Streptococcus in pregnant women with penicillin allergy.J Reprod Med 2007;52(06):480-484

11 Shenoy ES, Macy E, Rowe T, Blumenthal KG. Evaluation and management of penicillin allergy: a review. JAMA 2019;321(02):188-199

12 Sampson HA, Muñoz-Furlong A, Campbell RL, et al. Second symposium on the definition and management of anaphylaxis: summary report-second National Institute of Allergy and Infectious Disease/Food Allergy and Anaphylaxis Network symposium. Ann Emerg Med 2006;47(04):373-380

13 Prematta T, Shah S, Ishmael FT. Physician approaches to betalactam use in patients with penicillin hypersensitivity. Allergy Asthma Proc 2012;33(02):145-151 